\title{
ADP-Ribosylation Factor Like GTPase 4C (ARL4C) augments stem-like traits of glioblastoma cells by upregulating ALDH1A3
}

\author{
Qian Chen ${ }^{1,3^{*}}$, Wen-juan $\mathrm{Fu}^{1 *}$, Xiao-peng Tang ${ }^{2}$, Lu Wang1, Qin Niu' ${ }^{1}$, Shuai Wang ${ }^{1}$, Yong Lin ${ }^{1}$, Mian-fu \\ $\mathrm{Cao}^{1}$, Rong $\mathrm{Hu}^{4}$, Hai-Yan Wen ${ }^{5}$, Yan Wang ${ }^{1}$, Xia Zhang ${ }^{1}$ and Xiao-Hong Yao ${ }^{1 凶}$ \\ 1. Institute of Pathology and Southwest Cancer Center, Southwest Hospital, Third Military Medical University (Army Medical University), and Key \\ Laboratory of Tumor Immunopathology, Ministry of Education of China, Chongqing, China. \\ 2. Department of Nephrology, Southwest Hospital, Third Military Medical University (Army Medical University), Chongqing, China. \\ 3. Cancer Center of Daping Hospital, Third Military Medical University (Army Medical University), Chongqing, China. \\ 4. Department of Neurosurgery, Southwest Hospital, Third Military Medical University (Army Medical University), Chongqing, China. \\ 5. Department of Pathology, the Affiliated Provincial Hospital, Anhui Medical University, Hefei, China. \\ *These authors contributed equally to this work.
}

$\triangle$ Corresponding author: Xiao-Hong Yao, Institute of Pathology and Southwest Cancer Center, Southwest Hospital, Third Military Medical University (Army Medical University), Chongqing, China. E-mail: yxh15@163.com.

(1) The author(s). This is an open access article distributed under the terms of the Creative Commons Attribution License (https://creativecommons.org/licenses/by/4.0/). See http://ivyspring.com/terms for full terms and conditions.

Received: 2020.02.18; Accepted: 2020.09.06; Published: 2021.01.01

\begin{abstract}
Glioma cells with stem cell-like properties are crucial for tumor initiation, progression and therapeutic resistance. Therefore, identifying specific factors in regulating stem-like traits is critical for the design of novel glioma therapeutics.

Herein, we reported that ADP-Ribosylation Factor Like GTPase 4C (ARL4C) was highly expressed in glioma stem-like cells (GSLCs). GSLCs, determined by the efficiency of sphere formation in vitro and tumor growth in vivo, was increased by overexpression of ARL4C. ARL4C induced the tumorigenesis through ALDHIA3. Analyses of 325 patient specimens showed that ARL4C was highly expressed in glioblastoma (GBM) as compared with lower grade gliomas. In addition, higher level ARL4C expression in glioma was correlated with poorer progression-free survival and overall survival of patients. Therefore, ARL4C may act as a novel prognostic marker and a therapeutic target for GBM.
\end{abstract}

Key words: ARL4C; glioblastoma; glioma stem-like cell; ALDH1A3

\section{Introduction}

Glioblastoma (GBM) is featured with heterogeneity, increased invasiveness, high recurrence rate and resistance to therapy, which were attributed to the presence of glioma stem-like cells (GSLCs) in tumors [1, 2] Therefore, it is important to identify novel approaches targeting GSLCs.

Small GTPases of Ras superfamily are composed of more than 100 members, which share a conserved structure and biochemical properties, acting as binary molecular switches turned on by binding GTP and off by hydrolyzing GTP to GDP [3]. The key function of Ras superfamily members is the regulation of actin cytoskeleton rearrangement, cell growth and sustaining cell stemness [4,5]. Small monomeric GTPases of Ras superfamily also include the most prominent druggable cancer targets [6], such as CDC42, RAC1 and RhoA, which are important for the growth of GBM cells [7]. However, the precise role of Ras superfamily proteins in affecting GSLC features remains unclear.

ARL4C, a member of Ras superfamily, is associated with migration, invasion and proliferation of cancer cells and is regarded as a therapeutic target in colorectal cancer, lung cancer [8] and liver cancer [9]. We previously reported that high level expression of ARL4C promoted the invasion of PTEN-deficient 
GBM cells [10]. In order to examine the clinical significance of ARL4C and its role in controlling GSLCs, we evaluated the contribution of ARL4C to sustaining the stemness of GSLCs and the underlying molecular mechanism. In addition, we analyzed the expression of ARL4C in clinical glioma specimens and its association with patient prognosis.

\section{Materials and Methods}

\section{Cell culture}

The human GBM cell lines U87-MG, T98G from the ATCC were cultured as described [10]. Human normal glial cell line HEB was generously provided by Professor Guang-mei Yan (Department of Pharmacology, Sun Yat-sen University, Guangzhou, China). Primary GBM cells (GBM-1) were obtained from GBM tumor tissues (Southwest Hospital, AMU, China) and cultured as previously described [11].

GSLCs derived from GBM cells (U87-MG and GBM-1) were cultured with Neurobasal medium (Life Technologies, Carlsbad, CA, USA) with EGF (20 ng/ml, PeproTech, Rocky Hill, NJ, USA), bFGF $(20 \mathrm{ng} / \mathrm{ml}$, PeproTech) and B27 Supplement (20 $\mathrm{\mu l} / \mathrm{ml}$, Life Technologies, Carlsbad, CA, USA).

\section{Patients and tissue specimens}

This study included three independent cohorts of glioma patients, who did not receive preoperative radiotherapy, chemotherapy or immunotherapy. In cohort of Chongqing, glioma tissue samples were collected during the years 2013 to 2015. In cohort of Hefei, samples were collected during the years 2007 to 2011. In the Shanghai cohort, glioma tissue microarrays were purchased from BioChip (Shanghai, China), which were collected during the years from 2008 to 2011. All patients were followed up for 5 years and clinic pathologic parameters were obtained from medical records maintained in the Departments of Pathology of respective hospitals (Supplementary Table 1). The study concerning human specimens were performed in accordance with the principles of the Helsinki Declaration and approved by the Ethics Committee of Third Military Medical University (Army Medical University), Chongqing, China and the Affiliated Provincial Hospital of Anhui Medical University, Hefei, China. Glioma diagnosis was based on the World Health Organization (WHO) Classification of central nervous system tumor (2016).

\section{Immunohistochemical staining}

Immunohistochemical staining was conducted as previously described [12]. Paraffin-embedded GBM tissues and xenografts were cut into $5 \mu \mathrm{m}$-thick sections using a Dako REAL Envision Detection System (Dako, Glostrup, Denmark) according to manufacturer's instructions. The slides were incubated with rabbit polyclonal anti-ARL4C (1:100; ab-122025, Abcam, Cambridge, UK) at $4^{\circ} \mathrm{C}$ overnight. After removing the primary antibody with PBS, a horseradish per-oxidase (HRP)-conjugated secondary antibody (Dako) was added and the slides were incubated at $37^{\circ} \mathrm{C}$ for $30 \mathrm{~min}$. Semi-quantitation of ARL4C was independently performed according to the staining intensity and the percentage of positive tumor cells as previously described [13]. The staining intensity was scored as follows: 0 , no staining; 1 , weak staining; 2, moderate staining; and 3, strong staining. The percentage of positive cancer cells was scored as: $1=1-25 \% ; 2=26-50 \% ; 3=51-75 \%$; and $4>75 \%$. All slides were evaluated independently by two pathologists without knowledge of the identity of patients and the clinical outcome.

\section{Western blotting and qPCR}

Western blotting was conducted as previously described [12]. Primary antibodies used were: antiARL4C (1:500, ab-122025, Abcam), anti-ALDH1A3 (1:500, ab-129815, Abcam), anti-OCT4 (1:1000, CST, Danvers, MA, USA) and anti-SOX2 (1:1000, CST, Danvers, MA, USA).

Total RNA was extracted from the cell lines with RNAiso reagent according to the manufacturer's protocol. Reverse-transcription and quantitative Realtime PCR were performed using a One Step SYBRPrimer: ARL4C F: 5-CCAGTCCCTGCATATCG T-CAT-3; R: 3-TTCACGAACTCGTTGAACTTGA-5; RAB26; F: 5-GTCTGCTGGTGC GATTCAAG-3; R: 3-GCATGGGTAACACTGC GGA-5; RAB27B F: 5-AACAAGGC AGACCTACCA GAT-3; R: 3-TTCC CCATCCAAGTTTCCAGA-5'; RAB6B: F: 5-TGTACG ACAGCTTCGACAACA-3; R: 3-CTGCGGAACCTCT CCTGAC-5.

\section{In vitro limiting dilution and sphere forming assays}

In vitro limiting dilution assay was performed as previously described [13] and analyzed with an online software (http://bioinf.wehi.edu.au/software/ elda/). The sphere with a diameter $>50 \mu \mathrm{m}$ was counted under microscopy.

\section{Lentiviral infection procedures}

For overexpression of ARL4C in GBM cells, full length human ARL4C were generated and inserted into a lentivirus vector. Lentiviral particles containing ARL4C were packaged. GBM-1 and U87 cells $\left(1 \times 10^{6} /\right.$ well $)$ were infected with lentivirus containing constructs. The cells were selected and enriched by 4 $\mu \mathrm{g} / \mathrm{ml}$ puromycin. 
GBM-1 and U87 cells $\left(1 \times 10^{6} /\right.$ well) were infected with shARL4C lentivirus or empty control lentivirus vectors (Genechem, Shanghai, China). Cells were selected and enriched by $4 \mu \mathrm{g} / \mathrm{ml}$ puromycin. The sequence for the shRNAs were listed in Supplementary Table 2 .

\section{cDNA microarray analysis}

Affymetrix Whole Human Genome Oligo Microarray analysis was conducted by Shanghai Genechem Corporation (Shanghai, China). Genes differentially expressed with logarithmic ratios exceeding 2 -folds were defined significantly $(p<0.05)$. The gene chip data on shARL4C-1 and shCtrl-GBM-1 were deposited under accession number GSE121253 in NCBI Gene Expression Omnibus (GEO).

\section{Tumor implantation}

Five-week-old female NOD-SCID mice were purchased from Laboratory Animal Center of Southwest Hospital, Third Military Medical University (Army Medical University) (Chongqing, China). pLVX-eGFP-linker-luciferase lentivirus transfected U87-MG cells $\left(1 \times 10^{5} / \mu \mathrm{l}\right)$ were injected intracranially into the right frontal lobes of the mice. Two or four weeks later, xenograft tumors were quantified by bioluminescence imaging using an In vivo Image System (IVIS) Spectrum. Tumor-bearing mice were sacrificed to collect tumors when the animals became moribund. Animal experiments were approved by the Institutional Animal Care and Use Committee of Southwest Hospital, Third Military Medical University (Army Medical University) in accordance with the Guide for the Care and Use of Laboratory Animals.

\section{Statistical analysis}

All experiments were performed at least three times and data were shown as the mean \pm SD. Unpaired two-group comparison and multiple comparisons were made with the Student t-test or one-way ANOVA. Animal survival was analyzed using the Kaplan-Meier method, with the log-rank test for comparison. X-tile software was used to determine the cutoff point of ARL4C expression in clinical sample analysis [14].

\section{Results}

\section{ARL4C is preferentially expressed in glioma stem-like cells}

As Ras-superfamily members have been linked with tumor initiation and progression, we aimed to identify specific small GTPases of the superfamily that may be expressed in glioma stem-like cells (GSLCs) and examine their functional significance in tumor formation and progression. Microarray profiles comparing GSLCs and non-stem tumor cells (NSTCs) isolated from primary human GBM (GBM-1) (Fig. 1A) showed that 4 out of 100 members in the Rassuperfamily (RAB26, RAB27B, RAB6B and ARL4C) were significantly up-regulated in GSLCs $(P<0.05)$ (Fig. 1B). QRT-PCR indicated that ARL4C was consistently increased in all tested GSLCs compared with matched NSTCs (Fig. 1C). Increased expression of ARL4C protein in GSLCs relative to NSTCs was then confirmed (Fig. 1D) and co-localized with the GSLC marker CD133 in human GBM tissues (Fig. 1E).

\section{ARL4C is required for the self-renewal and proliferation of GBM cell in vitro and in vivo}

We found ARL4C was highly expressed in GBM cells as compared with normal brain glial cells (Supplementary Fig. 1A). We then overexpressed or knocked down ARL4C in GBM cells and found decreased sphere-forming efficiency of shARL4C GBM cells as compared with shCtrl cells (Fig. 2A). In contrast, overexpressing ARL4C in GBM cells increased their sphere-forming capabilities (Fig. 2B and Supplementary Fig. 1B, 1C). Fig. 2C showed that expression of SOX2 decreased in shARL4C GBM cells. However, overexpression of ARL4C increased SOX2 in tumor cells.

To further examine the effects of ARL4C on tumorigenesis of GSLCs in vivo, we transplanted shARL4C and shCtrl GBM cells into the brain of NOD-SCID mice. Bioluminescent imaging detected tumors formed by shARL4C U87 cells with smaller volumes compared to those formed by shCtrl U87 cells (Fig. 2D). Mice bearing tumors formed by shARL4C U87 cells showed longer survival than those transplanted with shCtrl U87 cells (Fig.2E). These data suggested that ARL4C was crucial for the tumorigenesis of GBM cells.

\section{The ARL4C/ALDH1A3 axis facilitated the tumorigenesis of GBM cells}

Microarray analyses were then performed to compare shCtrl-GBM-1 with shARL4C-1-GBM-1 cells for gene expression (Fig. 3A), ALDH1A3, a predominant ALDH enzyme that participates in diverse range of biological processes in glioma stem cells $[15,16]$, was most correlated to ARL4C expression in TCGA-LGGGBM database among 198 genes selected by comparison of differential gene expression in shCtrl- and shARL4C-1 GBM-1 cells (Fig. 3B). Western blotting showed that the expression of ALDH1A3 was higher in ARL4C-high GBM specimens than in ARL4C-low GBM specimens (Fig. 3C). Additionally, ALDH1A3 was decreased in shARL4C-1 GBM cells than that in shCtrl cells, and 
with an increase in OEARL4C GBM cells (Fig. 3D and 3E). When overexpressing ARL4C or knocking down of ALDH1A3 in U87 cells (Fig. 4A and 4B), we found that ARL4C increased the sphere forming ability of GBM cell, which is partially decreased by the knockdown of ALDH1A3. In examining the capacity of ALDH1A3 knockdown to reverse tumor growth, we found that knockdown of ALDH1A3 attenuated the pro-oncogene effects of ARL4C overexpressed in GBM cells (Fig. 4C and 4D). The survival of mice

\section{A}

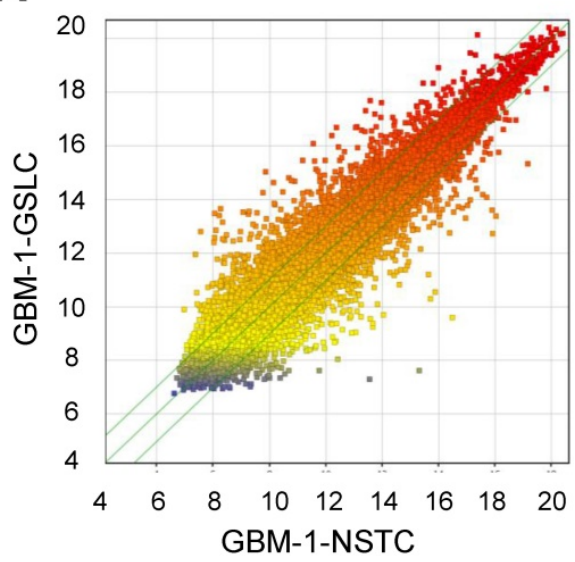

C

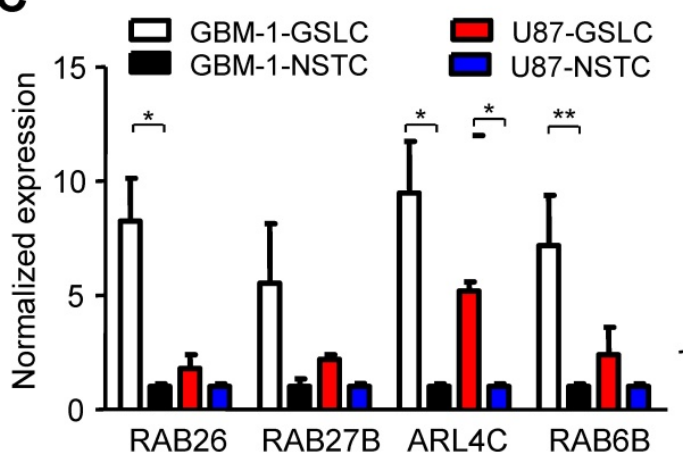

bearing-tumor formed by $\operatorname{sh} A L D H 1 A 3$ and OEARL4C-cells were markedly increased as compared to those bearing tumors formed by OEARL4C-cells alone (Fig. 4E). IHC staining of xenograft tumors showed that the expression of ALDH1A3 and SOX2 was decreased in tumors derived from shARL4C-1-GBM cells as compared with those derived from shCtrl-GBM cells (Fig. 4F). Thus, ALDH1A3 appears to be a target in ARL4C-mediated tumorigenic process.

B

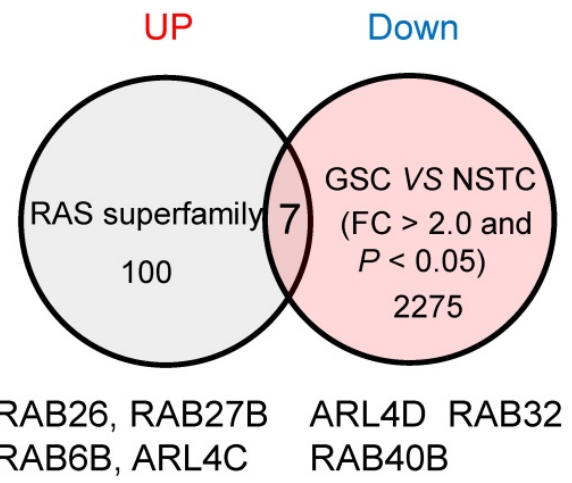

D

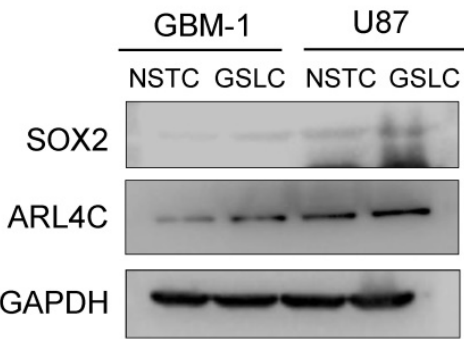

\section{$\mathbf{E}$}
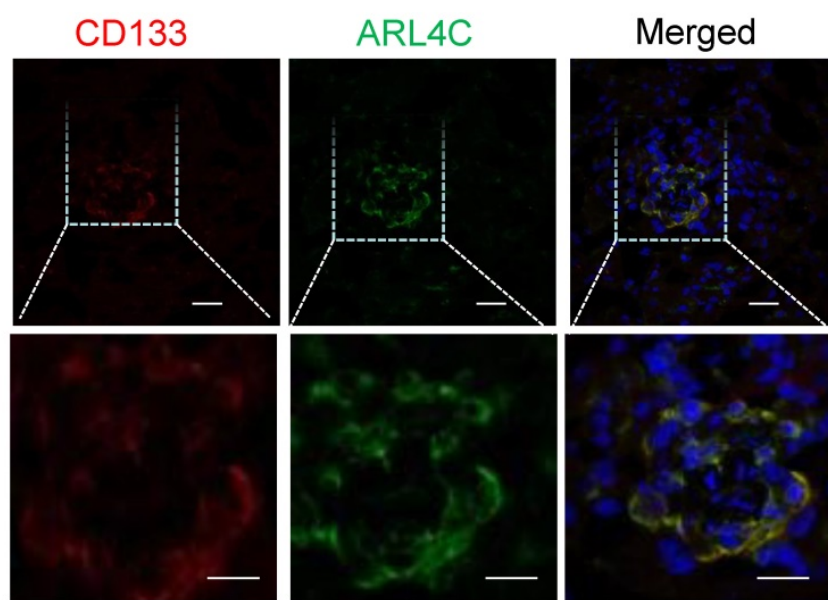

Figure 1. ARL4C is preferentially expressed in GSLCs. (A) Scattered map of gene expression in GSLCs relative to NSTCs from genechip. A total of 2275 genes were identified with $>2.0$ fold changes. (B) Seven genes were screened by comparison of Ras superfamily sets. Four candidates, RAB26, RAB27B, RAB6B, and ARL4C were significantly upregulated in GSLCs. (C) QRT-PCR analyses showing the expressions of RAB26, RAB27B, RAB6B, and ARL4D in GSLCs $(n=2)$ relative to matched NSTCs $(n=2)$. (D) Western blotting indicating the preferential expression of ARL4C and the GSLC marker SOX2 in GSLCs $(n=6)$ as compared with NSTCs. (e) ARL4C colocalized with CD133 in frozen GBM tissues. Scale bar $=20 \mu \mathrm{m}$; CD133: red; ARL4C: green; nucleus: blue. 

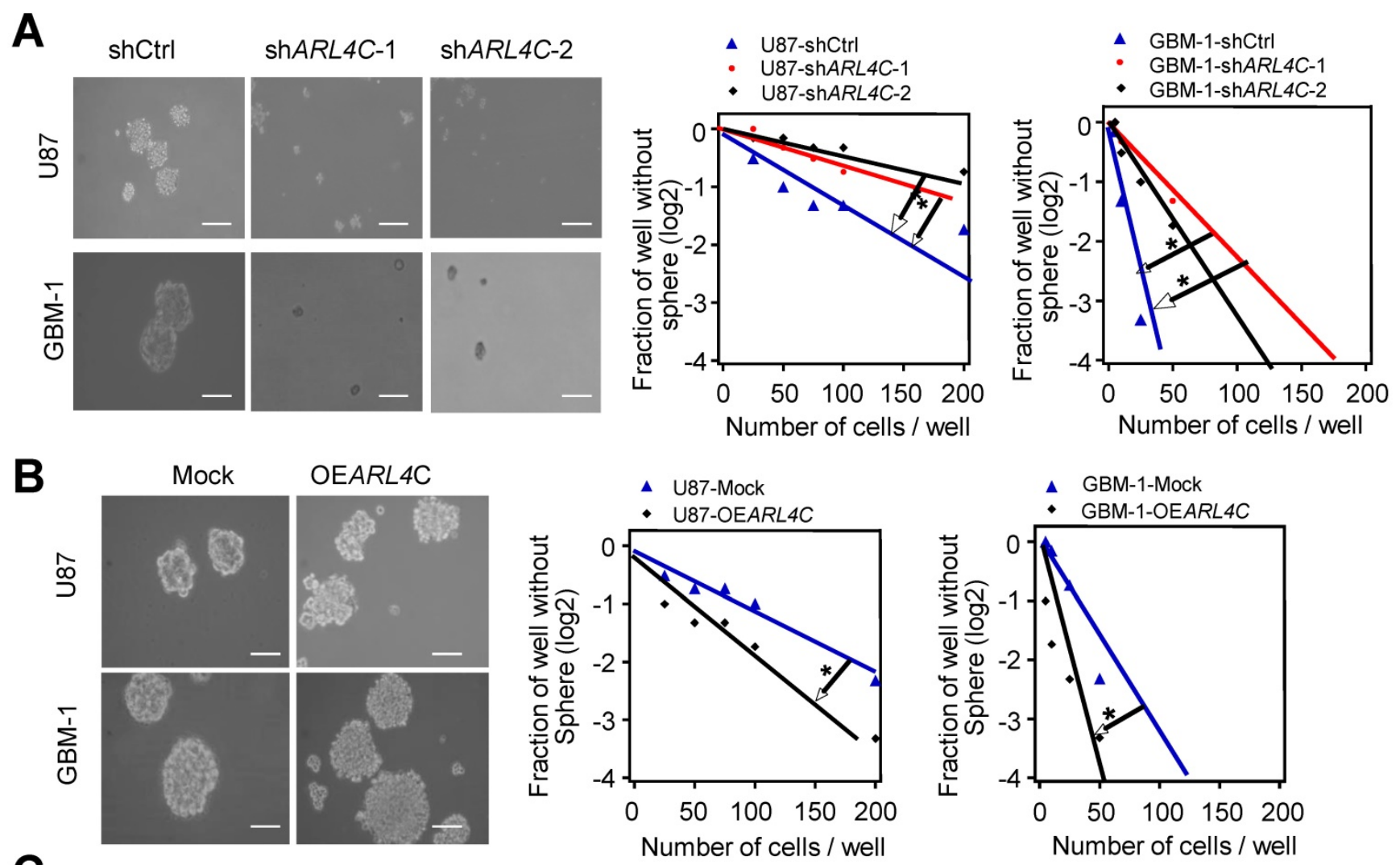

C
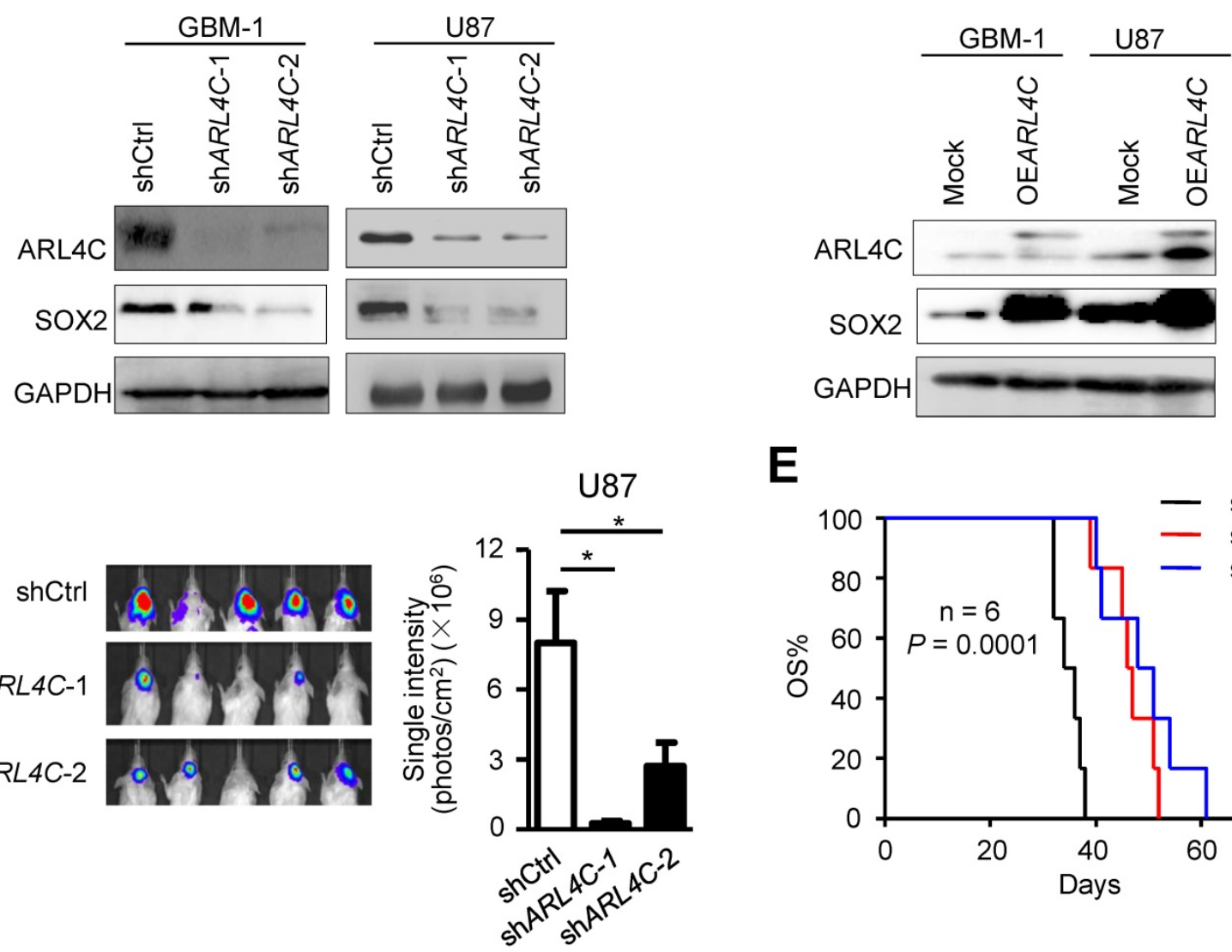

$\mathbf{E}$

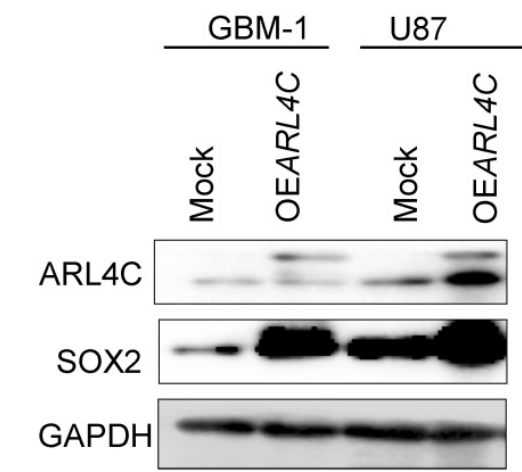

D

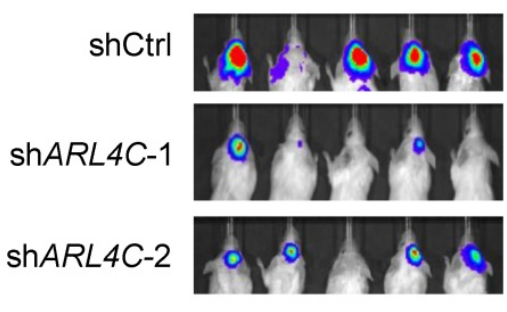

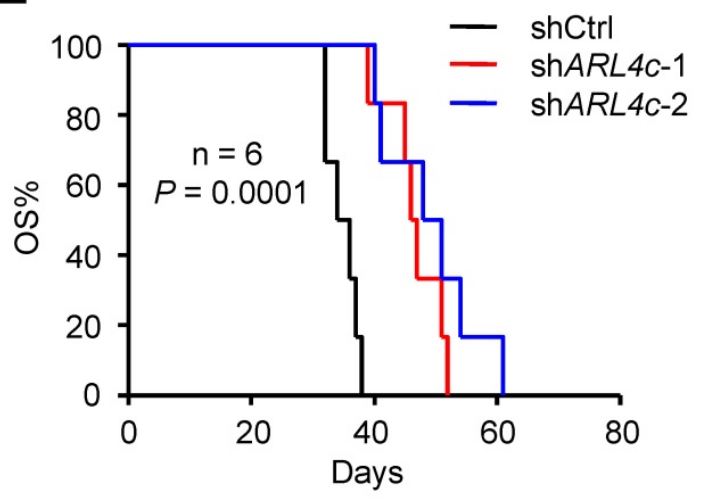

Figure 2. ARL4C increases the stem-like traits of GBM cells in vitro and their tumorigenesis in vivo. (A) Representative images (Left panel) and sphere-forming efficiency (Right panel) of shARL4C-1, shARL4C-2 and shCtrl GBM cells. Scale bar $=100 \mu \mathrm{m}$. (B) Representative images (Left panel) and sphere-forming efficiency (Right panel) of OEARL4C GBM cells and Mock cells. Scale bar $=100 \mu \mathrm{m}$. (C-D) Western blotting of SOX2 in cell groups with down- (C) and upregulated -(D) ARL4C as compared with control cells. (E) Bioluminescence images (Left panel) and quantification (Right panel) of tumors in NOD-SCID mice implanted with shCtrl, shARL4C-I-and shARL4C-2 U87 cells. (F) Survival curves of tumor-bearing mice implanted with shCtrl, shARL4C1 and shARL4C2 U87 cells $(* P<0.05 ; * * P<0.01)$. 
A
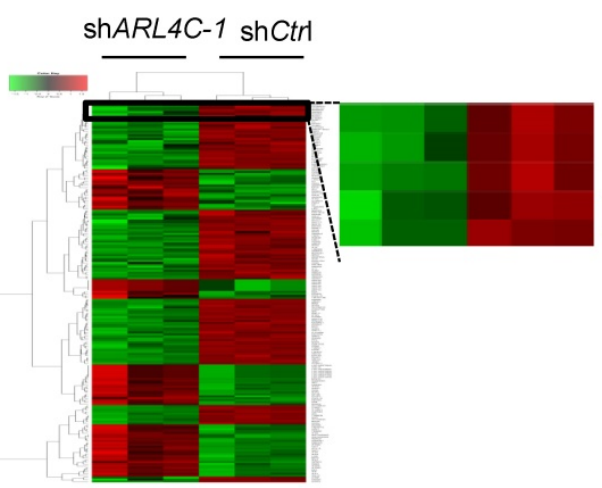

AGFG1

CSRP2

ZC3HAV1L

ALDH1A3

RMND5A

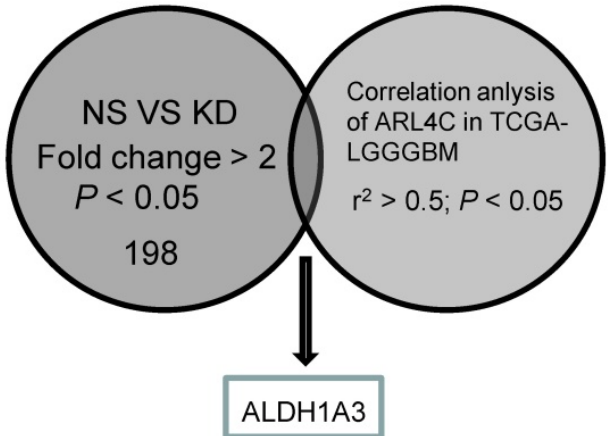

ALDH1A3
C

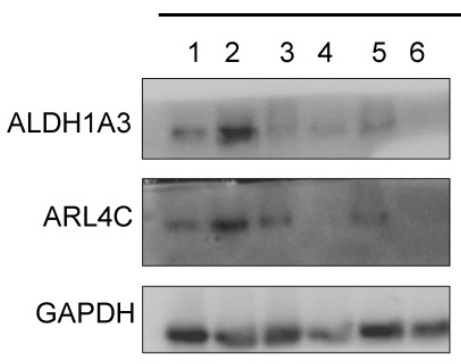

D
GBM case
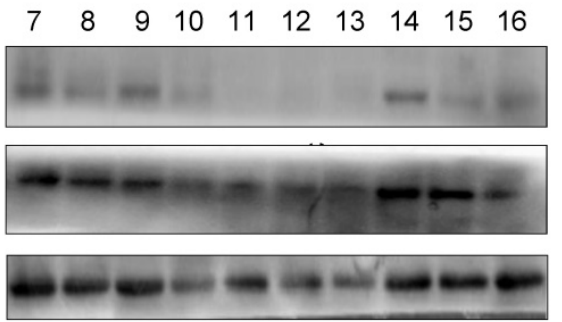

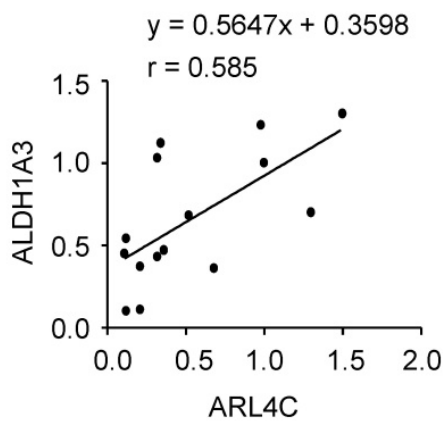

E

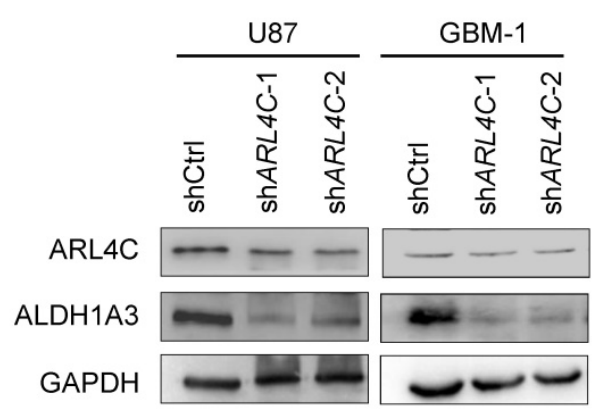

Figure 3. ARL4C augments the tumorigenesis of glioma cells by upregulating ALDH1A3 expression. (A) Heatmap depicting 198 transcripts differentially expressed in shCtrl and shARL4Cl GBM cells as indicated by $>2.0$ fold changes. (B) ALDHIA3 gene identified by interacting with differential gene sets and correlation analysis of ARL4C expression in TCGA-LGGGBM database as indicated by $\mathrm{r}^{2}>0.5$. (C) Western blotting (Right panel) and statistical analysis (Left panel) of ARL4C and ALDH1A3 expression in 16 human GBM tissues. (D) Western blotting of ALDHIA3 in shARL4C-1-, shARL4C-2-, and shCtrl GBM cells. (E) Western blotting of ALDH1A3 in Mock and OEARL4C GBM cells.

\section{ARL4C was associated to poor prognosis in patients with high grade glioma}

Validating the relationship between ARL4C expression and human glioma malignancy revealed that human gliomas expressed relatively higher levels of ARL4C than corresponding normal brain tissues (Fig. 5A). In 325 patients with gliomas, ARL4C was markedly increased in high-grade tumors. Furthermore, ARL4C expression in Grade IV GBM was higher than that in Grade III anaplastic gliomas $(P<0.05$; Fig. 5B). Similar results were obtained from 620 patients with gliomas from TCGA database (Fig. $5 C)$.

Kaplan-Meier analyses showed that patients with lower level of ARL4C had longer overall survival (OS; $P=0.0043$ ) and progression-free survival (PFS; $P$ $=0.0028)$ than those with higher level ARL4C in tumors. Similar results were obtained in all three regional-cohorts. Additionally, higher ARL4C expression was significantly correlated with poorer progression-free survival and overall survival of patients with Grade IV GBM (Supplementary Fig. 2A). In contrast, no correlation between ARL4C and patient survival was observed in patients with Grade $\Pi$ and Grade III tumors (Supplementary Fig. 2B and 2C). Therefore, higher ARL4C expression was associated with poorer prognosis in gliomas and ARL4C serves as a predictor. 


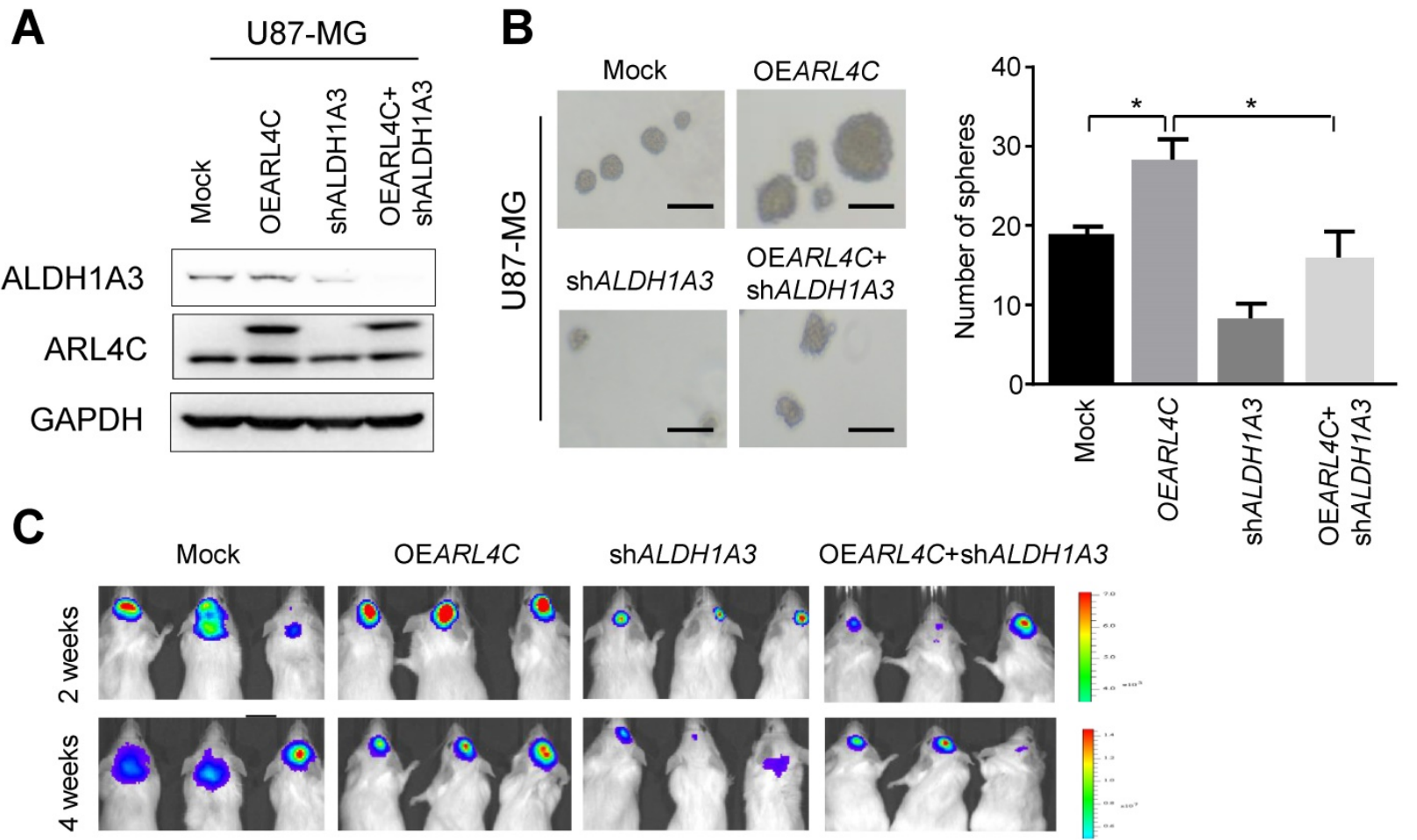

D

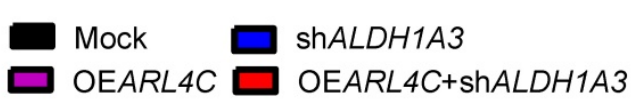

E - 1-Mock - 3-ShALDH1A3

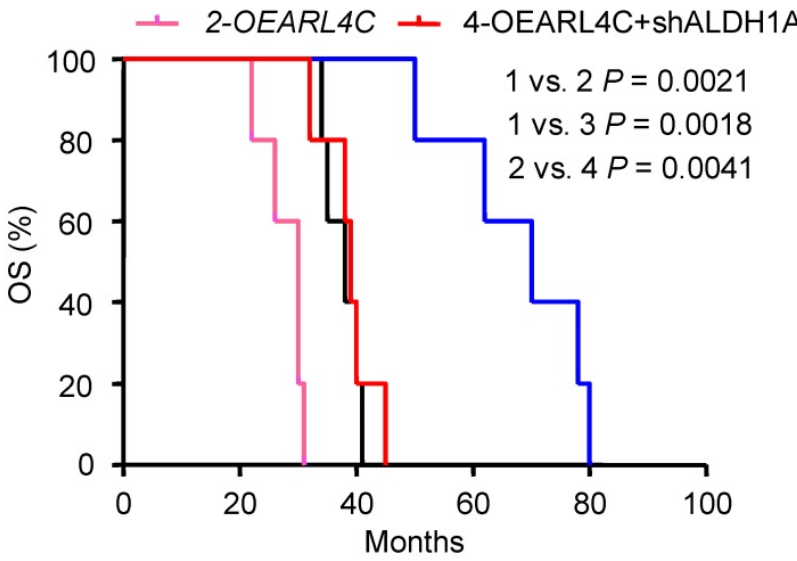

vs. $2 P=0.0021$

1 vs. $3 P=0.0018$

$\mathbf{F}$

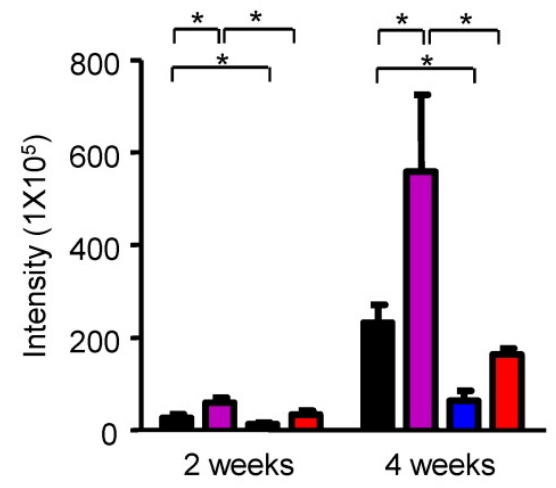

SOX2

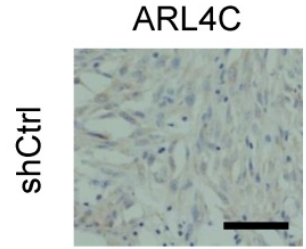

ALDH1A3
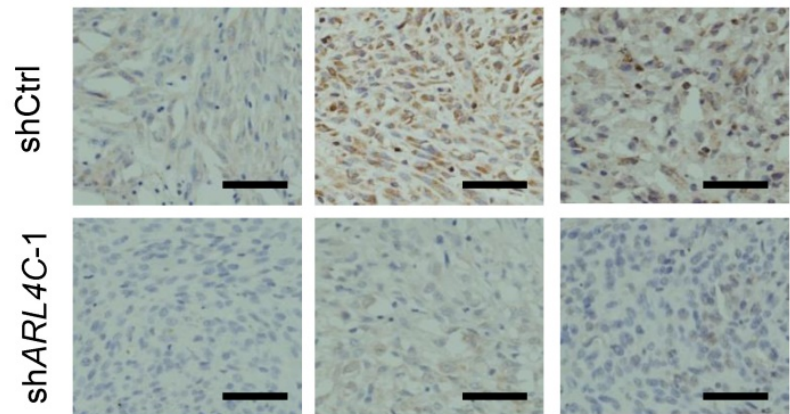

Figure 4. ARL4C/ALDHIA3 promotes the progression of GBM xenografts in vitro and vivo. (A) Immunoblotting of $A L D H 1 A 3$ and $A R L 4 C$ in $U 87$ cells overexpressing ARL4C (OEARL4C), ALDHIA3 knocking down (shALDHIA3), OEARL4C + shALDHIA3 and control vectors (Mock) cells. (B) Representative images (Left panel) and sphere-forming efficiency (Right panel) of mock, OEARL4C and shALDHIA3, OEARL4C + shALDHIA3 GBM cells. Scale bar = $100 \mu$ m. (C-D) Representative bioluminescence images (C) and quantification (D) of 2- and 4- weeks xenograft tumors formed by U87 cells overexpressing ARL4C (OEARL4C), ALDHIA3 knocking down (shALDHIA3), OEARL4C + shALDHIA3 and control vectors (Mock) cells. (E) Kaplan-Meier survival analysis of mice bearing tumors formed by shARL4C, shALDHIA3, OEARL4C + shALDHIA3 and Mock U87 cells. $* P<0.05$; $* * P<0.01$. (F) Representative IHC staining for ARL4C, ALDH1A3 and SOX2 in tumors formed by shCtrl and shARL4C-1 GBM cells. Scale bar $=50$ $\mu \mathrm{m}$. 


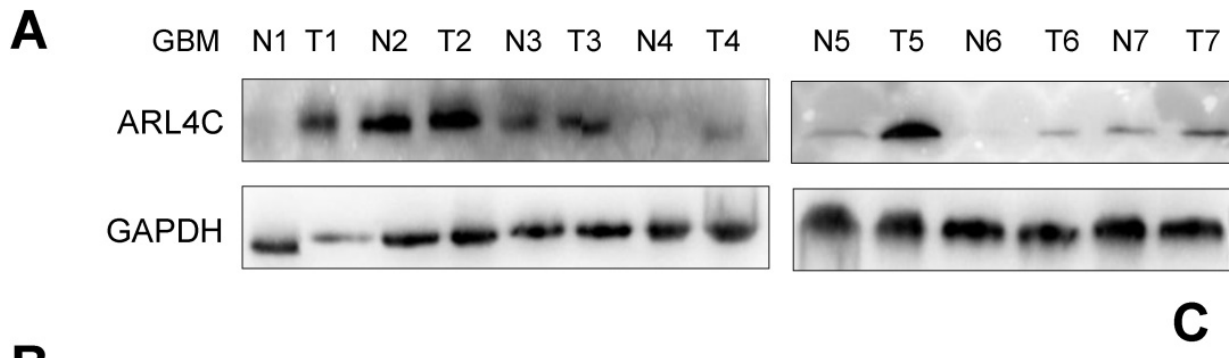

B

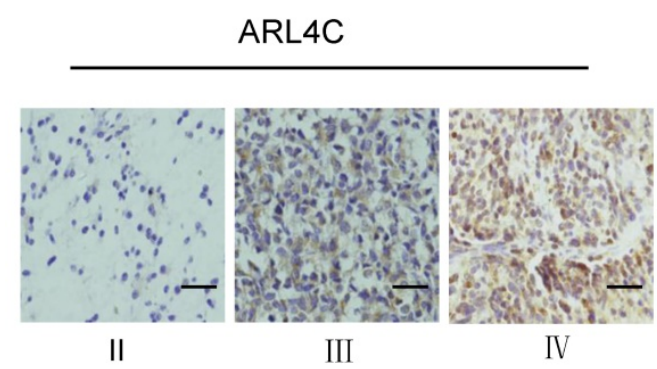

D

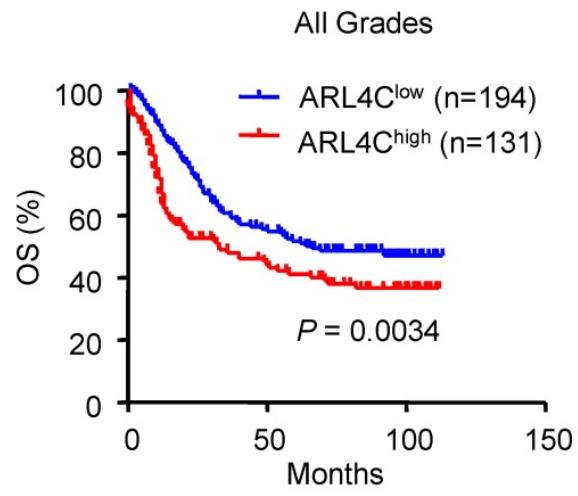

Clinical Specimen Cohort
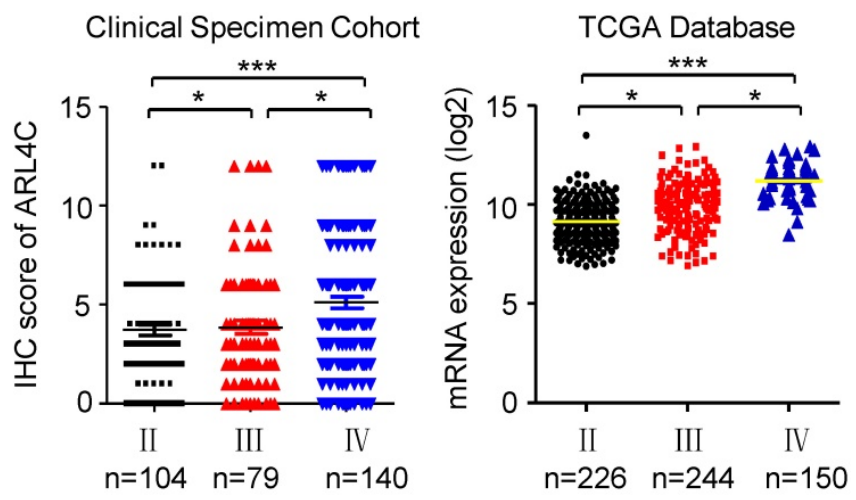

E
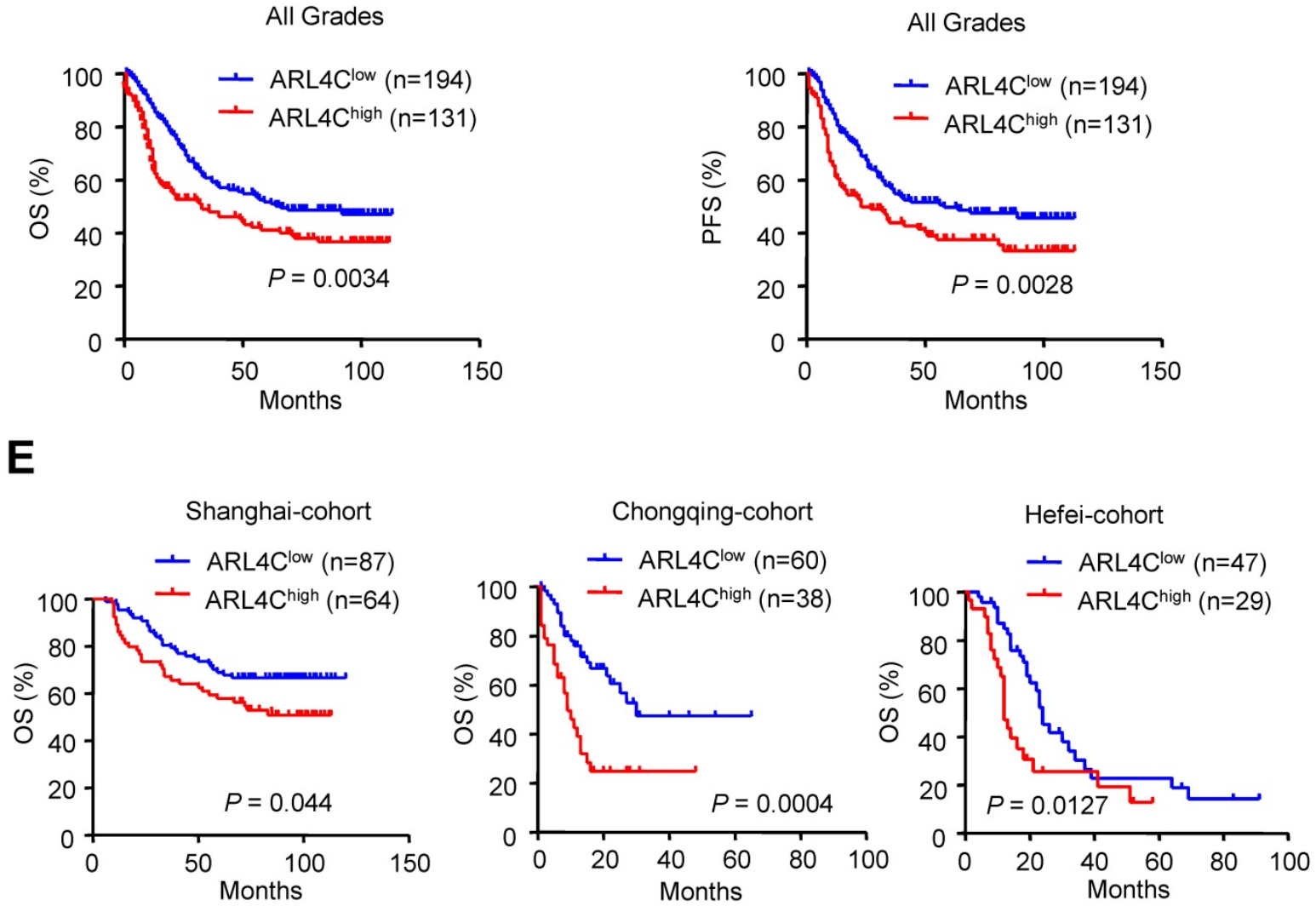

Figure 5. ARL4C is highly expressed in human gliomas and correlated with poor prognosis. (A) Western blotting showing the expression of ARL4C in seven pairs of glioma tumor tissues $(\mathrm{T})$ and corresponding adjacent normal tissues $(\mathrm{N})$. (B) Representative images (Left panel) and IHC score (Right panel) of ARL4C staining in tumors from patients with different grade gliomas. Scare bar $=50 \mu \mathrm{m}$. (C) ARL4C expression in tumors with different grades from TCGA-database. (D) Overall survival and progression free survival rate of patients in low versus high ARL4C expressing gliomas. (E) Kaplan-Meier curves of the overall survival rate of glioma patients with high versus low ARL4C expressing tumors in Shanghai, Chongqing and HeFei.

\section{Discussion}

Glioma stem like cells (GSLCs), a subset of tumor cells with stem cell characteristics such as the preferential expression of stem cell markers and the enhanced self-renewal ability, are crucial for tumorigenesis, progression and control of the plasticity of tumor cells $[17,18]$. Given the distinct gene 
sets and signaling pathways differentially expressed in gliomas with different grades, GSLCs in each grade may contain diverse and dysregulated pathways that govern unique phenotype of tumor growth, progression, and resistance to therapy $[19,20]$. Our current studies found that ARL4C was highly expressed in GSLCs and contributed to their stemness. We also showed that ARL4C was associated with tumor grade and poorer OS of patients, suggesting that ARL4C acts as an oncoprotein in glioma.

ARL4C, a Ras-GTP binding protein, was involved in various tumor progression processes $[21,22]$. The expression of ARL4C is regulated by many factors and pathways, such as promoter methylation, Wnt- $\beta$-catenin and growth factor-Ras signaling [23,24]. Our previous study found that PTEN/AKT/mTOR pathway stabilized ARL4C and decreased its ubiquitination. However, the downstream pathway of ARL4C was unknown. Here, we identified that ALDH1A3 as the main downstream target of ARL4C, and that ARL4C/ALDH1A3 axis was crucial for the tumorigenicity of glioma cells.

ALDH1A3 is a member of ALDH superfamily, which is vital for oxidizing endogenous and exogenous aldehydes [25]. Elevated ALDH has been considered a cancer stem cell marker in multiple tumor types [26]. Recent studies have shown that ALDH1A3 was required for sustaining ALDH activity in cancer stem cells [27] and was also regarded as a key determinant for the maintenance of mesochymal features of GSLCs. ALDH1A3 expression is regulated by several factors including promoter methylation, STAT3, hepatocyte growth factor (HGF)/c-MET pathways and protein ubiquitination. Our study defined ARL4C as a novel regulator that increases ALDH1A3 activity in GSLCs.

In summary, we provided evidence for ARL4CALDH1A3 axis in regulating GSLC properties and its value as a predictor of glioma patient survival.

\section{Supplementary Material}

Supplementary figures and tables.

http://www.jcancer.org/v12p0818s1.pdf

\section{Acknowledgments}

This study was supported by the grant cstc2018jcyj-yszxX0008, and the research project no. 82002656, no. 81874087 from the National Natural Science Foundation of China and no. 2017jsz110 from Key Laboratory of Tumor Immunopathology, Ministry of Education of China.

\section{Competing Interests}

The authors have declared that no competing interest exists.

\section{References}

1. Auffinger B, Spencer D, Pytel P, et al. The role of glioma stem cells in chemotherapy resistance and glioblastoma multiforme recurrence. Expert Rev Neurother. 2015; 15:741-52.

2. Gaelzer MM, Santos MSD, Coelho BP, et al. Hypoxic and reoxygenated microenvironment: stemness and differentiation state in glioblastoma. Mol Neurobiol. 2017; 54: 6261-72.

3. Goitre L, Trapani E, Trabalzini L, The Ras superfamily of small GTPases: the unlocked secrets. Methods Mol Biol. 2014; 1120:1-18.

4. Ridley A J, Paterson HF, Johnston CL, et al. The small GTP-binding protein rac regulates growth factor-induced membrane ruffling. Cell. 1992; 70: 401-410.

5. Cancelas JA. On how Rac controls hematopoietic stem cell activity. Transfusion. 2011; 51:153S-159S.

6. Zhao W, Jamshidiha M, Lanyon-Hogg T, et al. Direct targeting of the Ras GTPase superfamily through structure-based design. Curr Top Med Chem. 2017; 17:16-29

7. Fortin ES, Mathews IT, Symons MH, et al. Implications of Rho GTPase signaling in glioma cell invasion and tumor progression. Front Oncol. 2013; 3:241

8. Ahn T, Fergani C, Coolen LM, et al. Prenatal testosterone excess decreases neurokinin 3 receptor immunoreactivity within the arcuate nucleus KNDy cell population. J Neuroendocrinol. 2015; 27:100-110.

9. Harada T, Matsumoto S, Hirota S, et al. Chemically modified antisense oligonucleotide against ARL4C inhibits primary and metastatic liver tumor growth. Mol Cancer Ther. 2019; 18:602-612.

10. Chen $\mathrm{Q}$, Weng HY, Tang XP, et al. ARL4C stabilized by AKT/mTOR pathway promotes the invasion of PTEN-deficient primary human glioblastoma. J Pathol. 2019; 247:266-278.

11. Shi Y, Chen C, Yu SZ, et al. miR-663 suppresses oncogenic function of CXCR4 in glioblastoma. Clin Cancer Res. 2015; 21:4004-4013.

12. Shi Y, Chen C, Zhang X, et al. Primate-specific miR-663 functions as a tumor suppressor by targeting PIK3CD and predicts theprognosis of human glioblastoma, Clin. Cancer Res. 2014; 20:1803-1813.

13. Shi Y, Zhou WC, Cheng L, et al. Tetraspanin CD9 Stabilizes gp130 by Preventing Its Ubiquitin-Dependent Lysosomal Degradation to Promote STAT3 Activation in Glioma Stem Cells . Cell death differ. 2017; 24:167-180.

14. Camp RL, et al. X-tile: a new bio-informatics tool for biomarker assessment and outcome-based cut-point optimization. Clin Cancer Res. 2004; $10 \cdot 7252-7259$

15. Thomas ML, Antueno R, Coyle KM, et al. Citral reduces breast tumor growth by inhibiting the cancer stem cell marker ALDH1A3. Mol Oncol. 2016; 10:1485-1496.

16. Duan JJ, Cai J, Guo YF, et al. ALDH1A3, a metabolic target for cancer diagnosis and therapy. Int J Cancer. 2016; 139:965-975.

17. Wang $X$, Yang $K$, Xie $Q$, et al. Purine synthesis promotes maintenance of brain tumor initiating cells in glioma. Nat Neurosci. 2017; 20: 661-673.

18. Batlle E, Clevers H. Cancer stem cells revisited. Nat Med. 2017; 23:1124-1134.

19. Verhaak RG, Hoadley KA, Purdom E, et al. Integrated genomic analysis identifies clinically relevant subtypes of glioblastoma characterized by abnormalities in PDGFRA, IDH1, EGFR, and NF1. Cancer Cell. 2010; 17: 98-110.

20. Vescovi AL, Galli R, Reynolds BA. Brain tumour stem cells. Nat Rev Cancer. 2006; 6: 425-436.

21. Hu Q, Masuda T, Sato K, Taro Tobo, et al. Identification of ARL4C as a peritoneal dissemination-associated gene and its clinical significance in gastric cancer. Ann Surg Oncol. 2018; 25:745-753.

22. Harada T, Matsumoto S, Hirota $S$, et al. Chemically modified antisense oligonucleotide against ARL4C inhibits primary and metastatic liver tumor growth. Mol Cancer Ther. 2019; 18:602-612.

23. Fujii S, Shinjo K, Matsumoto S. Epigenetic upregulation of ARL4C, due to DNA hypomethylation in the 3'-untranslated region, promotes tumorigenesis of lung squamous cell carcinoma. Oncotarget. 2016; 7:1571-1587.

24. Matsumoto S, Fujii S, Kikuchi A. Arl4c is a key regulator of tubulogenesis and tumourigenesis as a target gene of Wnt-beta-catenin and growth factor-Ras signalling. J Biochem. 2017; 161:27-35.

25. Vasiliou V, Pappa A, Estey T. Role of human aldehyde dehydrogenases in endobiotic and xenobiotic metabolism. Drug Metab Rev. 2004; 36:279-299. [PubMed: 15237855].

26. Alison MR, Guppy NJ, Lim SM, et al. Finding cancer stem cells: are aldehyde dehydrogenases fit for purpose? J Pathol. 2010; 222:335-344.

27. Shao C, Sullivan JP, Girard L, et al. Essential role of aldehyde dehydrogenase 1A3 for the maintenance of non-small cell lung cancer stem cells is associated with the STAT3 pathway. Clin Cancer Res. 2014, 20:4154-4166. 